\title{
The James Webb Space Telescope
}

\author{
Jonathan P. Gardner ${ }^{1}$ \\ NASA's Goddard Space Flight Center \\ Laboratory for Observational Cosmology, Code 665 \\ Greenbelt MD 20771, USA \\ E-mail: jonathan.p.gardner@nasa.go
}

The scientific capabilities of the James Webb Space Telescope (JWST) fall into four themes. The End of the Dark Ages: First Light and Reionization theme seeks to identify the first luminous sources to form and to determine the ionization history of the universe. The Assembly of Galaxies theme seeks to determine how galaxies and the dark matter, gas, stars, metals, morphological structures, and active nuclei within them evolved from the epoch of reionization to the present. The Birth of Stars and Protoplanetary Systems theme seeks to unravel the birth and early evolution of stars, from infall onto dust-enshrouded protostars, to the genesis of planetary systems. The Planetary Systems and the Origins of Life theme seeks to determine the physical and chemical properties of planetary systems around nearby stars and of our own, and investigate the potential for life in those systems. To enable these four science themes, JWST will be a large $(6.6 \mathrm{~m})$ cold $(50 \mathrm{~K})$ telescope launched to the second Earth-Sun Lagrange point early in the next decade. It is the successor to the Hubble Space Telescope, and is a partnership of NASA, ESA and CSA. JWST will have four instruments: The Near-Infrared Camera, the Near-Infrared multi-object Spectrograph, and the Tunable Filter Imager will cover the wavelength range 0.6 to 5 microns, while the Mid-Infrared Instrument will do both imaging and spectroscopy from 5 to 28.5 microns. In this paper, I review the status and capabilities of the observatory and instruments in the context of the major scientific goals.

From planets to dark energy: the modern radio universe

University of Manchester, Manchester, UK

1-5 October, 2007

\footnotetext{
$1 \quad$ Speaker
} 


\section{The End of the Dark Ages: First Light and Reionization}

The James Webb Space Telescope (JWST; Gardner et al. 2006) seeks to identify the first luminous sources to form and to determine the ionization history of the early universe. The emergence of the first sources of light in the universe marks the end of the "Dark Ages" in cosmic history, a period characterized by the absence of discrete sources of light. Some time after the appearance of the first sources of light, hydrogen in the intergalactic medium was reionized. Results from the Wilkinson Microwave Anisotropy Probe combined with data on quasars at $\mathrm{z} \sim 6$ from the Sloan Digital Sky Survey show that this reionization had a complex history. Although there are indications that galaxies produced the majority of the ultraviolet radiation which caused the reionization, the contribution of quasars could be significant.

What Are the First Galaxies? To identify a sample of high redshift galaxies, JWST will make an ultra-deep imaging survey using several broad-band filters. The Lyman break technique will identify objects at increasing redshifts up to $\mathrm{z}=30$ or higher. For dwarf galaxies with $10^{6} \mathrm{M}_{\odot}$ of zero-metallicity massive stars at $15<\mathrm{z}<30$, the expected AB magnitude at emitted wavelengths just longward of Lyman $\alpha$ is $\sim 31$ mag. This ultra-deep survey will allow the derivation of galaxy counts as a function of redshift, seeing a drop at the formation of the first sources. The intensity of the non-ionizing continuum can be calibrated to yield the star formation rate as a function of redshift. The deep broad-band imaging in the near infrared will be enable follow-up low-resolution spectroscopy and mid-infrared imaging of promising sources. The imaging could be timed to search for supernovae.

When and How Did Reionization Occur? JWST will obtain high signal-to-noise, $\mathrm{R}=$ 1000 , near-infrared spectra of high-redshift QSOs or galaxies in order to determine the presence of a Gunn-Peterson trough or of a Lyman $\alpha$ damping wing (Figure 1; X. Fan, private communication). The targets will be the brightest known high redshifts objects, perhaps found in a wide-area Spitzer warm mission survey (Gardner et al. 2007). A damping wing should be present for a few million years, before the ionizing radiation is sufficient to create a large Strömgren sphere around each ionizing source. In order to measure the ionizing continuum of a class of sources, we will measure their hydrogen and helium Balmer lines. Comparison between these lines gives an estimate for the steepness, or hardness of the ionizing continuum, providing a measurement of the rate of production of ionizing photons for any given class of sources under the assumption that the escape fraction is known. Identification of the nature of ionizing sources requires a combination of diagnostics: line shapes, line widths, line ratios, and the shape of the continuum. We expect the intrinsic line shapes and widths of AGN-powered sources to be broader than those of sources ionized by stellar radiation.

\section{The Assembly of Galaxies}

JWST seeks to determine how galaxies and the dark matter, gas, stars, metals, morphological structures, and active nuclei within them evolved from the epoch of reionization to the present day. Theory and observation show that galaxies are assembled through a process of the hierarchical merging of dark matter concentrations, accompanied by chemical evolution 


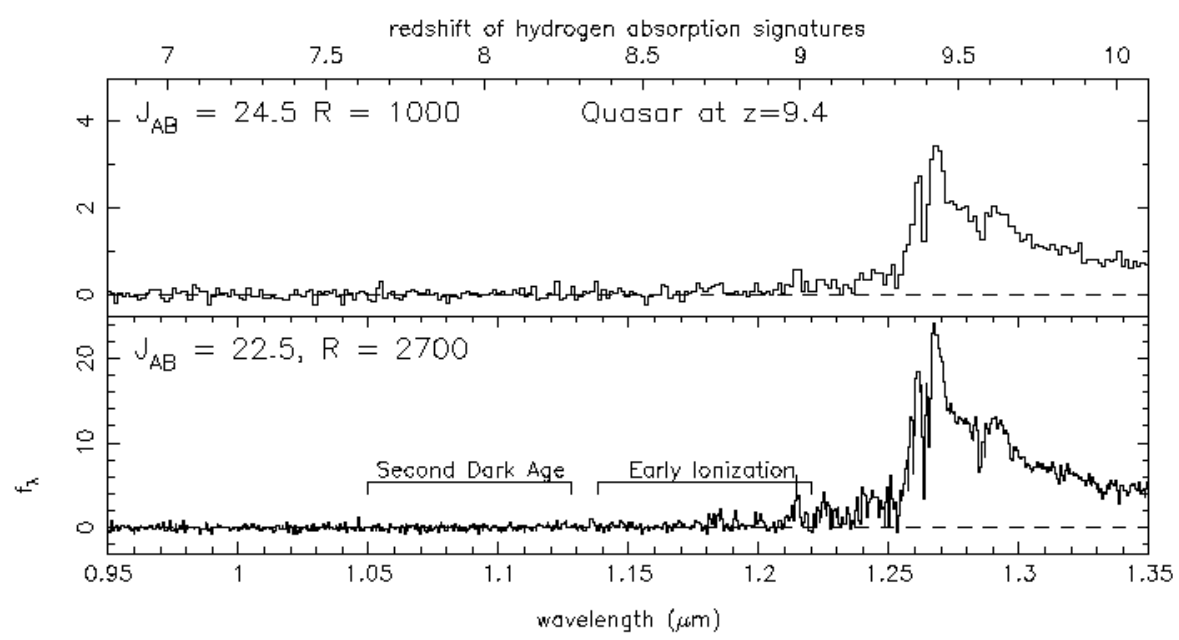

Figure 1. A simulated spectrum of a $z=9.4$ quasar observed with JWST's NIRSpec for 300 ksec. In the top figure, a faint quasar is observed with spectral resolution $\mathrm{R}=1000$, while in the bottom figure a brighter quasar is observed at higher resolution. The simulation includes an early reionization, followed by a recombination and a second dark age. (X. Fan, private communciation.)

of the gas and dust, and resulting in the Hubble Sequence of galaxies. However, we do not really know how galaxies are formed, what controls their shapes, and what makes them form stars. We do not know how the chemical elements are generated and redistributed through the galaxies, and whether the central black holes exert great influence over the galaxies. We do not know the global effects of violent events as small and large parts join together in collisions.

How Did the Hubble Sequence and Heavy Elements Form? To trace the formation of the Hubble Sequence of galaxies, JWST will determine the morphologies, stellar populations, and star-formation rates in a very large sample of galaxies observed in deep-wide imaging and spectroscopic surveys, as a function of their mass, environment, and cosmic epoch. JWST will also determine when the long-lived stars in a typical galaxy were formed, whether in situ or in smaller galaxies that subsequently merged together to form a large galaxy. Direct characterization of the merging rate of galaxies will provide another angle on this question.

What Physical Processes Determine Galaxy Properties? Despite the variety of galaxy properties observed today, galaxies obey a number of remarkably tight scaling relations between basic properties of luminosity, size, kinematics and metal enrichment. These include the TullyFisher relation for disk galaxies and the "fundamental plane", and projections thereof, for spheroids. More recently, a surprising relationship between the mass of the central black hole and the properties of the surrounding spheroid (e.g., the velocity dispersion) has been established. JWST will investigate these processes through deep imaging for structural parameters plus high-resolution spectroscopy for kinematical data. The dark matter will be revealed through gravitational weak-lensing surveys reaching $\mathrm{z} \sim 2.5$. JWST will also use midinfrared spectroscopy to diagnose the energy sources in the interior of bright systems, as starbursts have strong PAH features, while AGN have strong [NeVI] $7.66 \mu \mathrm{m}$ lines. 


\section{The Birth of Stars and Protoplanetary Systems}

JWST seeks to unravel the birth and early evolution of stars, from infall on to dustenshrouded protostars, to the genesis of planetary systems. The formation of stars and planets is a complex process, even in the well-developed paradigm for a single, isolated low-mass star. Things are even more complicated, as stars very rarely form in isolation. The current picture of star formation starts on large scales, as molecular cloud cores cool and fragment to form highly dynamic clusters of protostars, spanning the mass spectrum from $\mathrm{O}$ stars to planetary-mass brown dwarfs. Within those clusters, individual young sources are often encircled by disks of warm gas and dust, where material aggregates to form proto-planetary systems. These disks are the source of highly-collimated jets and outflows, which transfer energy and angular momentum from the infalling material into the surrounding medium, and clear away the remainder of the birth core. On larger scales, the intense ultraviolet flux and strong winds of the most massive stars can disperse an entire molecular cloud, while simultaneously ionizing and evaporating the circumstellar disks of the surrounding lower-mass stars.

How do Clouds Collapse and form Protoplanetary Systems? Stars form in small $(\sim 0.1$ pc) regions undergoing gravitational collapse within larger molecular clouds. Standard theory predicts that these cores collapse from the inside out, but there are alternative theories which predict different density distributions in the cores. By measuring the extinction to background stars, JWST will map those density distributions in order to understand the relative roles that magnetic fields, turbulence, and rotation play while the clouds collapse to form stars. Once selfgravitating molecular cloud cores have formed, they collapse to form protostellar seeds, which gain material via continuing accretion. These protostars will begin radiating in the $10 \mu \mathrm{m}$ to 20 $\mu \mathrm{m}$ region, since radiation from the warm central source is scattered off dust grains in the inner envelope into the line-of-sight. Direct imaging of disks around low-mass stars reveal their internal density and temperature structure, and show how they are affected by their environment. Since circumstellar disks are both a product and a mediator of the star formation process, as well as the progenitors of planetary systems, it is clear that a fuller understanding of the evolution of circumstellar disks will play a key role in our understanding of these central topics. Spectra of circumstellar disks will trace the formation history of planetesimals and other solid bodies, their composition, their processing, and their possible future evolution.

How do Very High and Very Low-Mass Stars Form? The formation of massive stars produces intense winds and ionizing radiation which impacts the surrounding molecular cloud material and the nascent circumstellar disks of adjacent low-mass stars. The mechanism by which massive stars form is not yet known. The standard disk-accretion scenario appropriate for low-mass stars cannot be simply scaled up, since predictions are that the radiation pressure from the growing central source would build up so quickly that no more material could accrete, limiting the mass of the source. Likewise, the low-mass opacity-limited cutoff for star formation is predicted to be 3 to $10 \mathrm{M}_{\mathrm{JUP}}$, although a more detailed consideration of the role of magnetic fields may bring this down to $1 \mathrm{M}_{\mathrm{JUP}}$. JWST will conduct imaging and spectroscopic surveys of young star-forming regions to establish the both the high and low-mass limits of the initial mass function, and to determine the processes that operate at the extremes of star formation. 


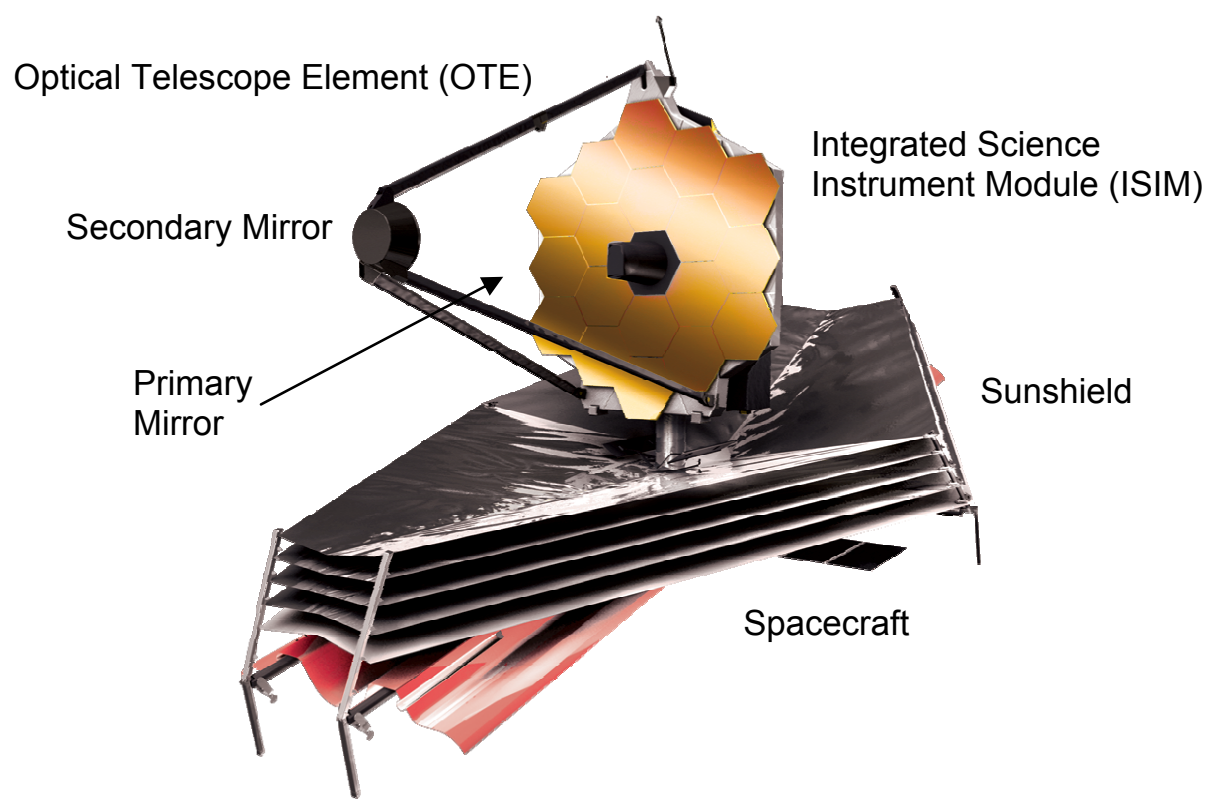

Figure 2. The JWST Observatory. The optical telescope element (OTE) contains the primary and secondary mirrors, the integrated science instrument module (ISIM) element contains the instrumentation and the spacecraft element consists of the spacecraft and the sunshield.

\section{Planetary Systems and the Origins of Life}

JWST seeks to determine the physical and chemical properties of planetary systems including our own, and to investigate the potential for the origins of life in those systems.

How Do Planets Form? The formation of multiple objects is a common outcome of star formation, including binary or higher-order star systems, a central star orbited by brown dwarfs and/or planets, or a star with a remnant disk of particulates. Brown dwarfs and giant planets might arise from two different formation mechanisms, or planets could be the low-mass tail of a single star-formation process. The formation of giant planets is a signpost, detectable with JWST, of a process that may also generate terrestrial planets. In contrast, direct collapse formation of brown dwarfs may signal systems in which terrestrial planet formation is rare or impossible, because of the required disk mass, angular momentum and subsequent disk evolution. JWST will conduct coronagraphic surveys of nearby stars to find mature Jovian companions, and of more distant systems to find young planets. Spectroscopy of isolated or widely separated planets or sub-brown dwarfs will reveal basic physical parameters such as gravity, composition, temperature and the effect of clouds. The remnant of the circumstellar disk that formed our Solar System is observable today as the smaller planets, moons, asteroids and comets, along with the zodiacal light and interplanetary gas and dust. JWST spectroscopy of comets will reveal how circumstellar disks are like our own Solar System.

How Are Habitable Zones Established? Some geochemical evidence suggests that Earth's water did not come from locally formed planetesimals. The source of water is uncertain; it could have been delivered through impacts of asteroids and comets, although isotopic ratios call this into question. By measuring isotopic ratios in comets and larger Kuiper Belt bodies, JWST can solve this part of the puzzle, removing a major uncertainty in the source of water for 


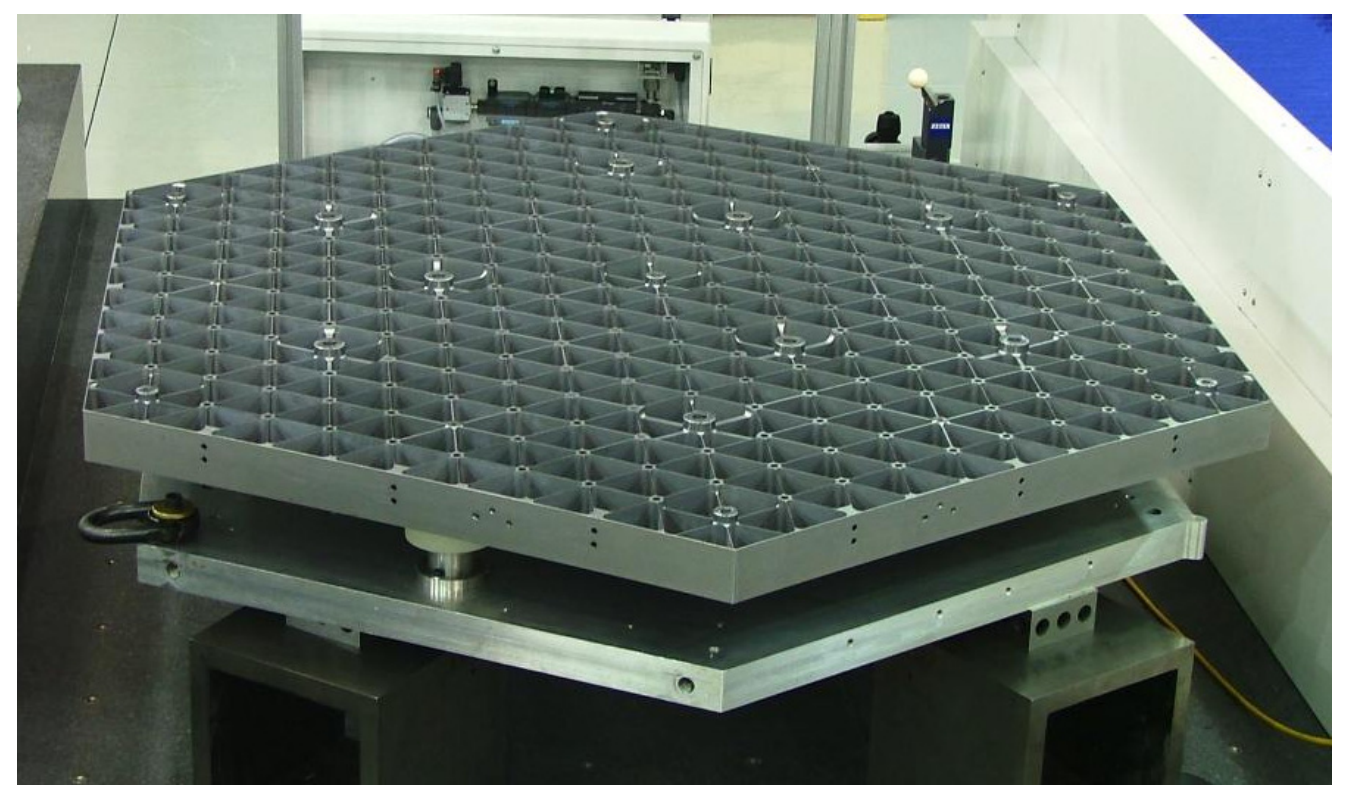

Figure 3. A primary mirror segment, currently in the polishing phase.

our own planet. JWST will also monitor icy bodies in the outer Solar System, such as Titan, in order to investigate chemical processes in the early Solar System.

\section{JWST Implementation}

The JWST Project is organized into three segments: observatory, ground and launch. The observatory is composed of an optical telescope element (OTE), an integrated science instrument module (ISIM) containing the scientific instruments, a spacecraft, and a sunshield (Figure 2).

Observatory: The primary mirror is made up of 18 beryllium segments (Figure 3), each positioned by actuators with 6 degrees of freedom plus radius of curvature adjustment. The $f / 20$ three-mirror anastigmat telescope has a $25 \mathrm{~m}^{2}$ collecting area, and delivers a diffraction-limited Strehl ratio $>0.80$ at 2 microns and longer wavelengths. Most broad-band imaging observations at $<10 \mu \mathrm{m}$ wavelengths will have zodiacal background-limited sensitivity. The sunshield will passively cool the telescope to below $50 \mathrm{~K}$, with the NIR instruments at 37 to $40 \mathrm{~K}$. The MIRI will be actively cooled with a cryo-cooler to $7 \mathrm{~K}$. The field of regard for the telescope will enable all regions of the sky to be viewed during the year, and a continuous viewing zone within 5 degrees of each ecliptic pole will be available. JWST will be able to find a guide star $95 \%$ of the time for any available target at any available roll angle. The observational efficiency is designed such that $70 \%$ of the time the observatory will be available to make science observations, although this will depend on the particular program selected (and the consequent number of slews). The WFS\&C subsystem aligns the mirror segments on-orbit so that their wavefronts match properly, creating a diffraction-limited $6.5-\mathrm{m}$ telescope, rather than overlapping images from 18 individual $1.3 \mathrm{~m}$ telescopes. Determination of the wavefront error and the necessary OTE mirror commands is done on the ground using images taken in and out of focus. 
Instrumentation: The integrated science instrument (ISIM) module contains the science instruments for the observatory, and the support electronics for the science instruments: NIRCam, NIRSpec, TFI, and MIRI. A cryo-cooler will be used for cooling MIRI and its Si:As detectors. The near-infrared detector arrays in the other instruments are passively cooled $\mathrm{HgCdTe}$. In addition to the science instruments, the ISIM contains the fine guidance sensor (FGS) and the computer that directs the daily science observations based on plans received from the ground. Simultaneous operation of all science instruments is possible; this capability will be used for parallel calibration, including darks and possibly sky flats.

Near-Infrared Camera NIRCam provides filter imaging in the 0.6 to $5.0 \mu \mathrm{m}$ range with high sensitivity, wavelength multiplexing, and wide field of view. NIRCam consists of two fully redundant, identical optical trains mounted on two beryllium benches, each split with a dichroic at $2.4 \mu \mathrm{m}$. The detectors are eight $2 \mathrm{~K}$ arrays sampling the PSF with 32 mas pixels in the short wavelength channel and two $2 \mathrm{~K}$ arrays with 65 mas pixels at long wavelengths, delivering a simultaneous $2.2 \times 4.4$ arcmin field of view. Coronagraphic imaging is possible using a set of spots in the focal plane and Lyot stops in the pupil wheel. Current models predict a coronagraphic contrast of $\sim 10^{4}$ at 0.5 arcsec at a wavelength of $4.6 \mu \mathrm{m}$.

Tunable Filter Imager The tunable filter imager (TFI) provides narrow-band imaging in the wavelength ranges $1.9<\lambda<4.8$ over a field of view of $2.2 \times 2.2 \mathrm{arcmin}^{2}$ with a spectral resolution $\mathrm{R} \sim 100$. The TFI uses dielectric-coated Fabry-Perot etalon plates with a small air (vacuum) gap. The TFI incorporates four coronagraphic occulting spots permanently on one side of the field of view. The coronagraph will deliver a contrast ratio of $\sim 10^{4}(10 \sigma)$ at $1 \operatorname{arcsec}$ separation. The sensitivity is limited by speckle noise. Contrast ratios of $10^{5}$ may be achievable at sub-arcsec scales using roll or spectral deconvolution techniques.

Mid-Infrared Instrument The Mid-Infrared Instrument (MIRI; Figure 4) provides imaging and spectroscopic measurements within the wavelength range 5 to $29 \mu \mathrm{m}$. The imaging module provides broad-band imaging, coronagraphy, and low-resolution ( $\mathrm{R} \sim 100,5$ to $10 \mu \mathrm{m}$ ) slit spectroscopy using a single $1024 \times 1024$ pixel Si:As sensor chip assembly (SCA) with 110 mas pixels, giving an imaging field of view of $84 \times 113$ arcsec. MIRI imaging includes three coronagraphic phase masks for a quadrant-phase coronagraph and one opaque spot for a Lyot coronagraph. The MIRI integral field spectrograph uses dichroics and image slicers to obtain simultaneous $\mathrm{R} \sim 3000$ spectral and spatial data on a field of view that varies between 3 and 7 arcsec on a side. MIRI spectroscopy uses two 1K Si:As detector arrays.

Launch, Deployment and Orbit: JWST will be launched on an Arianespace Ariane 5 ECA rocket into orbit about the second Lagrange (L2) point, approximately $1.5 \times 10^{6} \mathrm{~km}$ from the Earth in the Earth-Sun system. The total observatory mass is $6500 \mathrm{~kg}$, including stationkeeping propellant sized for 10 years of operations. The observatory has the following five deployments: (1) deploy spacecraft bus appendages, (2) deploy sunshield, (3) extend telescope tower, (4) deploy secondary mirror support structure, and (5) deploy primary mirror wings. The deployment mechanism design includes heaters and other protections that eliminate the need for 


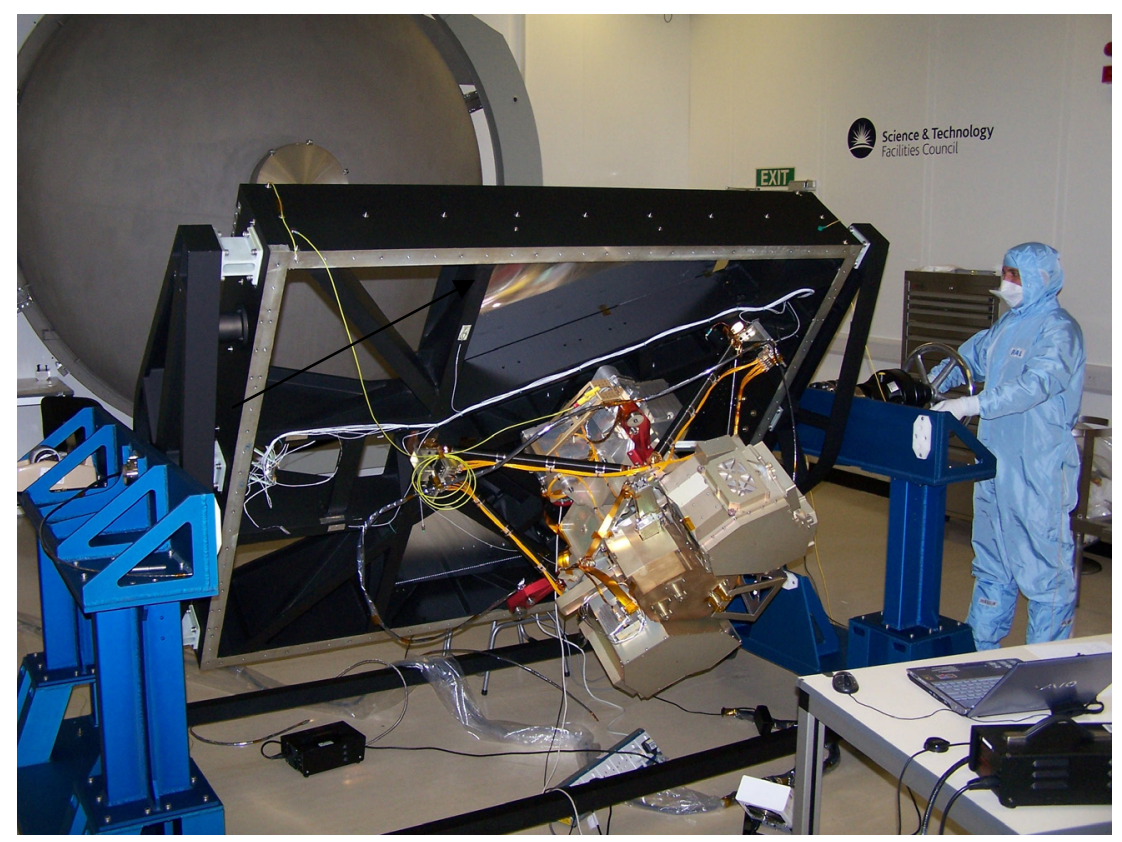

Figure 4. The Mid-Infrared Instrument (MIRI) verification model.

time critical events and allow for unlatching and re-latching to relieve any residual long-term stress in the structure.

Operations: JWST will be operated from a Science and Operations Center (S\&OC) located at the Space Telescope Science Institute, the organization that operates HST for NASA. Although the capabilities of JWST are being developed to address science themes discussed in the previous sections, about $85 \%$ of the observing time on JWST will be awarded through a series of proposal solicitations, which will be open to any astronomer in the world. The scope of JWST's competitively-selected investigations will range from large legacy-style projects, which last months and address a range of science goals simultaneously, to small programs that target important but very specific science objectives. The observatory will be operated through an event-driven architecture, with very little real-time commanding. Observation plan uplinks will be weekly, while data downlinks will be daily, using the Deep Space Network. The STScI will pipeline calibrate the data and distribute them to the astronomers through the World Wide Web.

\section{References}

[1] J. P. Gardner, et al. 2006, “The James Webb Space Telescope,” Space Science Reviews 123/4 (485)

[2] J. P. Gardner, X. Fan., G. Wilson, \& M. Stiavelli, 2007, “A Spitzer Warm Mission Ultra-Wide Survey as a Target Finder for the James Webb Space Telescope," in, The Science Opportunities of the Warm Spitzer Mission Workshop, ed. L. J. Storrie-Lombardi \& N. A. Silberman, (College Park: American Institute of Physics), in press. 